integrates whole genome sequencing, transcriptome sequencing (RNA-seq) and methylation array to provide an integrated multiomics pipeline, aiming to improve treatment outcomes for highrisk paediatric cancer patients $(<30 \%$ survival). Unique to ZERO, we have developed a comprehensive RNA-seq pipeline to accurately identify molecular aberrations, infer their predicted function and pathogenicity, and identify aberrantly expressed genes. Expression data is utilised to identify over-/underexpressed genes, interpret gain-of-function and loss-of-function mutations, resolve the impact of splice altering mutations on the transcriptome, and identify the expressed fusion products and the associated activated retained protein domains (such as tyrosine kinase domains) that are directly targetable.

From 2017-2021, ZERO was available across the full range of high-risk paediatric cancer subtypes. This integrated pipeline has identified the molecular basis for the cancer in almost all cases (94\%), with potentially actionable treatment recommendations being made for $72 \%$ of patients. Over 700 children have been enrolled on the national clinical trial to date, and ZERO is expanding the program to be available to all Australian children and young people diagnosed with cancer by the end of 2023 .

\section{MAKING SENSE OF RNA SPLICING VARIANTS}

Sandra T. Cooper $^{1,2,3}$

${ }^{1}$ Kids Neuroscience Centre, Kids Research, Children's Hospital at Westmead, Sydney, NSW, Australia; ${ }^{2}$ Discipline of Child and Adolescent Health, Faculty of Health and Medicine, University of Sydney, Sydney, NSW, Australia; ${ }^{3}$ The Children's Medical Research Institute, Westmead, Sydney, NSW, Australia

Determining clinically whether a coding or non-coding variant will actually disrupt pre-mRNA splicing and cause a genetic disorder is one of the grand challenges currently facing genetic pathology. Variant curation is a time-consuming use of highly skilled labour and the rate-limiting step for laboratory throughput. Splicing variant interpretation compounds these issues, exacerbated by the exponential growth of genomic testing. This talk will highlight advances in machine-learning and evidence-based approaches to predict if a DNA will disrupt mRNA splicing, and if so, how - exactly? Leveraging confirmed diagnostic and clinical outcomes for $\sim 100$ families triaged in real-time into research-led RNA diagnostic testing, we will discuss: strengths and weaknesses of different modes of RNA diagnostic testing; retrospective evaluation of the predictive accuracy of artificial intelligence splicing prediction tools; and, the clinical utility of empirical methods to reliably predict the nature of mis-splicing for pathology use of PVS1 or PP3/BP4, and/or, to direct strategic design of RNA Diagnostic assays. We will conclude with progress made by the Australian Consortium for RNA Diagnostics (SpliceACORD) with the singular vision to devise standardised practices, quality standards, and a robust, pragmatic and flexible health services implementation strategy to advance RNA diagnostics into clinical practice.

\section{MAKING SENSE OF P53 ISOFORMS}

Kelly A. Avery-Kiejda ${ }^{1,2}$

${ }^{1}$ School of Biomedical Sciences and Pharmacy, College of Health, Medicine and Wellbeing, The University of Newcastle,
Callaghan, NSW, Australia; ${ }^{2}$ Hunter Medical Research Institute, New Lambton Heights, NSW, Australia

The tumour suppressor, p53, has been classified as a 'guardian of the genome', due to its ability to coordinate multiple signalling pathways involved in the DNA damage response including the induction of cell cycle arrest and DNA repair or apoptosis. The importance of p53 in tumour suppression has long been established, with mutations arising in more than half of all human cancers. p53 has clear prognostic power where its somatic mutation is associated with tumour progression, resistance to therapy and poor prognosis. The complexity of p53 signalling has become increasingly apparent in recent years. p53 is now known to be expressed as 12 isoforms: p53 (fulllength), p53 $\beta, \quad \mathrm{p} 53 \mathrm{~g}, \mathrm{D} 133 \mathrm{p} 53, \mathrm{D} 133 \mathrm{p} 53 \beta, \mathrm{D} 133 \mathrm{p} 53 \mathrm{~g}$, D40p53, D40p53 $\beta$, D40p53g, D160p53, D160p53 $\beta$ and D160p53g; that can modulate its functional activity (positively and negatively). However, a detailed understanding of how they contribute to 553 function and cancer prognosis is still lacking. This presentation will outline our recent advances in understanding the function of p53 isoforms and their potential as biomarkers for the management of cancer, with an emphasis on breast cancer.

\section{DE NOVO DESIGN OF CRISPR-CAS13B ENABLES EFFICIENT SILENCING OF SARS-COV-2 VARIANTS AND TUMOUR PATHOGENIC RNAS WITH NEAR SINGLE-BASE PRECISION}

Wenxin $\mathrm{Hu}^{1,2}$, Wei Zhao ${ }^{3}$, Amit Kumar ${ }^{1,2}$, Gurjeet Jagjeet Singh ${ }^{1,2}$, Shijiao Qi ${ }^{1,2}$, Josh Casan ${ }^{1,2}$, Carolyn Shembery ${ }^{1,2}$, Danielle Fong ${ }^{3}$, Jennifer M. Zerbato ${ }^{3}$, Jori Symons $^{3}$, Rajeev Rudraraju ${ }^{3}$, Damian Purcell ${ }^{3}$, Michelle Haber $^{5}$, Ilia Voskoboinik ${ }^{1,2}$, Paul G. Ekert ${ }^{1,2,4,5}$, Sharon Lewin ${ }^{3}$, Joseph Trapani ${ }^{1,2}$, Mohamed Fareh ${ }^{1,2}$ ${ }^{1}$ The Sir Peter MacCallum Department of Oncology, University of Melbourne, Parkville, Vic, Australia; ${ }^{2}$ Cancer Immunology Program, Peter MacCallum Cancer Centre, Melbourne, Vic, Australia; ${ }^{3}$ The Department of Infectious Diseases, The University of Melbourne at the Peter Doherty Institute for Infection and Immunity, Melbourne, Vic, Australia; ${ }^{4}$ Murdoch Children's Research Institute, Parkville, Vic, Australia;

${ }^{5}$ Children's Cancer Institute, Randwick, NSW, Australia

Cas13 is a new reprogrammable CRISPR effector that targets single-stranded RNAs. We recently demonstrated efficient suppression of SARS-CoV-2 variants with reprogrammed Cas 13b. ${ }^{1}$ However, the poor understanding of Cas13b targeting mechanisms restricts its use for broader therapeutic applications.

To uncover these molecular bases, we developed innovative library screens in mammalian cells where we tested $>200$ singlebase resolution tiled CRISPR RNAs (crRNAs) targeting several transcripts. Remarkably, we revealed previously unknown RNA motifs within the spacer that are either highly enriched or depleted in extremely potent and unproductive crRNAs, respectively. We found a unique sequence at the 5 ' end of the spacer that greatly enhances Cas $13 \mathrm{~b}$ potency. We designed de novo crRNAs harbouring unnatural RNA motifs that we identified in the screen, which exhibited enhanced potency and largely outperformed conventional crRNAs. 
Finally, we leveraged these molecular features to reprogram Cas13b to silence several gene fusion transcripts that drive a variety of tumours. We show that targeting the breakpoint of fusion transcripts with de novo designed tiled crRNAs yields very high and specific silencing, with absolute discrimination between tumour-associated fusion transcripts and the wild-type variants expressed in normal cells.

Taken together, this study provides a molecular blueprint for Cas13b reprogramming against pathogenic transcripts.

\section{Reference}

1. Fareh M, Zhao W, Hu W, et al. Reprogrammed CRISPRCas13b suppresses SARS-CoV-2 replication and circumvents its mutational escape through mismatch tolerance. Nat Commun 2021; 12: 4270.

\section{RETINAL GENE THERAPIES}

Tom Edwards

Centre for Eye Research Australia, The Royal Victorian Eye and Ear Hospital, Melbourne, Vic, Australia

The eye is uniquely suited to gene-based therapies designed to target degenerative disease affecting the retina. These include progressive Mendelian inherited single gene disorders that lead to retinal degeneration and severe visual impairment. Inherited retinal disease is the most common cause of visual impairment in working aged Australians and is largely incurable. A number of gene-based therapies have reached clinical trial phase, with one granted TGA approval in 2021. Whilst this is certainly an exciting time for clinicians and patients alike, many challenges remain. This presentation will summarise the current status of retinal gene therapy, the surgical technique for gene delivery and future directions.

\section{USING EQA DATA TO DETERMINE RISK IN MOLECULAR DIAGNOSTIC TESTS}

\author{
$\underline{\text { Sze Yee Chai }}{ }^{1}$, Nalishia Munusamy ${ }^{1}$, Bruce Bennetts ${ }^{2}$, \\ Tony Badrick \\ ${ }^{1}$ Molecular Genetics, Royal College of Pathologists of \\ Australasia Quality Assurance Programs (RCPAQAP), Sydney, \\ NSW, Australia; ${ }^{2}$ Department of Molecular Genetics, The \\ Children's Hospital at Westmead, Sydney, NSW, Australia
}

Genetic pathology is a rapidly evolving field that plays a crucial part in diagnostic, prognostic and treatment decisions that directly influence the management of patients. Molecular testing errors resulting in false-negative or false-positive results can therefore be harmful to patients. To ensure that patients receive reliable results, participation in external quality assurance (EQA) programs is both an ISO requirement and essential to guarantee optimal clinical testing and reporting. The purpose of EQA programs includes continuous evaluation and monitoring of laboratory performance for specific tests, identification of interlaboratory differences, evaluation of method performances, and the harmonisation and standardisation of testing and reporting to improve results comparability. Presently, the RCPAQAP offers EQA programs mainly to clinical laboratories within the Australasia and Asia regions amongst a number of EQA providers based in Europe and the United States. A review of the 2020-2021 laboratory performance in the RCPAQAP
Molecular Genetics EQA programs, and the potential risks to patients, will be discussed.

\section{THE AUSTRALIAN GENOMICS CLINICAL, DIAGNOSTIC AND RESEARCH NETWORK}

John Christodoulou $^{1}$, Hamish Scott ${ }^{2}$, Julie McGaughran ${ }^{3}$

${ }^{1}$ Australian Genomics, Murdoch Children's Research Institute, The University of Melbourne, Melbourne, Vic, Australia; ${ }^{2}$ Department of Genetics and Molecular Pathology, Centre for Cancer Biology, An SA Pathology and UniSA alliance, SA Pathology, Adelaide, SA, Australia; ${ }^{3}$ Genetic Health Queensland, Metro North Health, The University of Queensland, Brisbane, Qld, Australia

Australian Genomics is an initiative that supports nationally funded genomic research projects. In doing so, Australian Genomics brings together key stakeholders into networks, with the express goal of using the outcomes of this research to help shape the equitable and robust delivery of clinical genomics in the Australian healthcare context.

The Clinical, Diagnostic and Research (CDR) Network is one of these key networks, with a multidisciplinary group over 80 genomics researchers, diagnosticians and healthcare professionals from across Australia. The frequency of the meetings for its stakeholders offers a more rapid, diverse and discursive communication forum than the various annual meetings such as Path update and HGSA, helping the genomics community keep abreast of the latest international and nationally relevant developments in our rapidly moving field.

The network meets regularly to discuss genomics initiatives, issues and opportunities, and to share the latest advancements in the field. Guest speakers present at each meeting on genomics initiatives, research and technological advances. The network also provides a forum for Australian Genomics and external stakeholders to engage the nation's genomics experts in initiatives of national and international significance, with recent examples including the consultation response to the Pricing Framework for Australian Public Hospital Services, consultation responses to MSAC applications, and Australian Genomics' business continuity plan.

A key challenge is ensuring that Australian Genomics can offer its support and acquired expertise to the genomics community beyond the life of the current grant. To this end, consultation is underway to develop an enduring continuity plan.

\section{OPTICAL GENOME MAPPING USING BIONANO: A COMPARATIVE STUDY OF GENOMIC CHANGES IN HAEMATOLOGICAL MALIGNANCIES PERFORMED AT THE JOHN HUNTER HOSPITAL}

$\underline{\text { Katie A. Ashton }}^{1}$, Nadine K. Berry ${ }^{1}$, Ashleigh Fodeades ${ }^{1}$, $\overline{\text { Raewyn Billings }}^{1}$, Susan Dooley ${ }^{2}$, Eva Chan ${ }^{2}$, Cliff Meldrum ${ }^{2}$, Kristen Palmer ${ }^{2}$, Andrew Harland ${ }^{3}$, Andrew Ziolkowski ${ }^{1}$, Anoop K. Enjeti ${ }^{1,4,5}$, Rodney J. Scott ${ }^{1,2,4}$

${ }^{1}$ Department of Molecular Medicine, NSW Health Pathology, John Hunter Hospital, NSW, Australia; ${ }^{2}$ Statewide Services/ Genomics, NSW Health Pathology, Newcastle, NSW, Australia; ${ }^{3}$ ICT Services, NSW Health Pathology, John Hunter Hospital, NSW, Australia; ${ }^{4}$ University of Newcastle, NSW, Australia; ${ }^{5}$ Calvary Mater Hospital, Newcastle, NSW, Australia 